\title{
Association of PTTG1 expression with invasiveness of non-functioning pituitary adenomas
}

\author{
Su Jung Kum, Hye Won Lee, Soon Gu Kim, Hyungsik Park, Ilseon Hwang, Sang Pyo Kim \\ Department of Pathology, Keimyung University Dongsan Medical Center, Daegu, Korea
}

\begin{abstract}
Background: Pituitary tumor transforming gene 1 (PTTG1), paired-like homeodomain 2 (PITX2), and galectin-3 have been widely studied as predictive biomarkers for various tumors and are involved in tumorigenesis and tumor progression. We evaluated the usefulness of PTTG1, PITX2, and galectin-3 as predictive biomarkers for invasive non-functioning pituitary adenomas (NFPAs) by determining the relationship between the expressions of these three proteins and the invasiveness of the NFPAs. We also investigated whether PTTG1, E-cadherin, and Ki-67, which are known to be related to each other, show a correlation with NFPA features. Methods: A retrospective study was conducted on 87 patients with NPFAs who underwent surgical removal. The NFPAs were classified into three groups based on magnetic resonance imaging findings of suprasellar extension and cavernous sinus invasion. Immunohistochemical staining for PTTG1, PITX2, galectin-3, E-cadherin, and Ki-67 was performed on tissue microarrays. Results: PTTG1 expression showed a statistically significant correlation with the invasiveness of NFPAs, whereas PITX2 and galectin-3 did not have a relationship with the invasiveness of NFPAs. Moreover, there was no association among PTTG1, E-cadherin, and Ki-67 expression. Conclusions: PTTG1 has the potential to serve as a predictive biomarker for invasive NFPA. Furthermore, this study may serve as a reference for the development of PTTG1-targeted therapeutic agents.
\end{abstract}

Key Words: Non-functioning pituitary adenomas; Pituitary tumor transforming gene expression; Invasiveness

Received: July 30, 2021 Revised: August 25, 2021 Accepted: August 31, 2021

Corresponding Author: Sang Pyo Kim, MD, PhD, Department of Pathology, Keimyung University Dongsan Hospital, Keimyung University School of Medicine,1095 Dalgubeol-daero, Dalseo-gu, Daegu 42601, Korea

Tel: +82-53-258-7398, Fax: +82-53-258-7382, E-mail: smkim5@kmu.ac.kr

Corresponding Author: Ilseon Hwang, MD, PhD, Department of Pathology, Keimyung University Dongsan Hospital, Keimyung University School of Medicine,1095 Dalgubeol-daero, Dalseo-gu, Daegu 42601, Korea

Tel: +82-53-258-7396, Fax: +82-53-258-7382, E-mail: ilseon@dsmc.or.kr

Pituitary adenoma is a common neuroendocrine tumor that accounts for approximately $17 \%$ of all primary intracranial neoplasms [1]. While most of these tumors are benign, some show aggressive patterns such as invasion into surrounding structures. In contrast to the functioning pituitary adenomas, which are usually quickly detected due to symptoms of excess hormone secretion, the detection of a non-functioning pituitary adenoma (NFPA) is relatively delayed. As a result, NFPAs are usually found as macroadenomas $(1-4 \mathrm{~cm})$ or giant adenomas $(>4 \mathrm{~cm})$ with suprasellar extension (SSE), which tend to invade into the cavernous sinus. The effectiveness of hormone control therapy for NFPA is limited, and surgical removal is the only effective treatment [2,3]. Based on these aspects, it is clinically important to identify prognostic markers of NFPAs.

In the 2004 World Health Organization (WHO) classification, three subcategories were proposed for the classification of primary pituitary tumors: typical, atypical, and carcinoma [4]. Atypical pituitary adenoma was diagnosed based on histopathological features, including a high Ki-67 proliferation index (>3\%), p53 expression, and a high mitotic count. However, as the WHO classification was revised in 2017, the term "atypical pituitary adenoma" is no longer recommended based on studies reporting that this subtype does not reflect prognosis [5-7]. Instead, histological or radiological invasiveness status has emerged as an important factor for predicting prognosis, which was also introduced in the 2017 WHO classification [5,8]. In general, two factors are predominantly used to evaluate the invasiveness status of pituitary adenoma: SSE and cavernous sinus invasion (CSI). CSI is directly associated with prognosis [9]. On the other hand, there are many studies indicating that SSE alone lacks prognostic 
value [10-12]. However, several grading systems combining SSE and CSI have shown significant prognostic value and are currently widely used [13].

Until the term "atypical pituitary adenoma" was accepted, p53 was generally used as a biomarker for aggressive pituitary adeno$\mathrm{ma}$ [14]. However, as various studies asserting that $\mathrm{p} 53$ is not suitable as a prognostic marker have been published, there is now no biomarker of aggressive pituitary adenomas with proven validity $[9,15,16]$. Currently, only a few candidate proteins are being studied [17].

This study focused on the pituitary tumor transforming gene 1 (PTTG1), paired-like homeodomain 2 (PITX2), and galectin-3 proteins as predictive biological markers for invasive NFPAs. PTTG1 and PITX2 are not widely used markers, but it has been continually reported that these proteins are related to tumors and their aggressiveness in various organs. PTTG1 is an oncogene that participates in mitosis, DNA repair, angiogenesis, and cell proliferation [18-21]. PTTG1 is also known to induce tumor aggressiveness by being involved in epithelial-to-mesenchymal transition (EMT) and down-regulating E-cadherin expression $[19,22,23]$. PITX2, a member of the paired-like homeobox transcription factor family, regulates cell cycle regulators such as cyclin D1, cyclin D2, and c-Myc. Galectin-3 is a well-known prognostic and diagnostic marker in various organs that is involved in various biological processes, including cell growth, angiogenesis, cell adhesion, and tumor progression [24,25].

In this study, we aimed to determine the correlation between PTTG1, PITX2, and galectin-3 expression levels and to evaluate the various clinicopathologic characteristics of the NFPAs, including invasiveness status. Moreover, we evaluated the relationships among PTTG1, E-cadherin, and Ki-67 in NFPAs. This is the first study to investigate the biological significance of PTTG1, PITX2, and galectin-3 in Korean patients with NFPAs.

\section{MATERIALS AND METHODS}

\section{Patients and tissue specimens}

The archived specimens of 124 patients with NFPAs, obtained from 2000 to 2019 by surgical resection, including the transsphenoidal approach, at Keimyung University Dongsan Hospital (Daegu, Korea), were analyzed in this study. A pathological diagnosis of the surgical specimens was made based on hematoxylin and eosin staining and immunohistochemical staining for six pituitary hormones (growth hormone, thyroid-stimulating hormone, prolactin, follicle-stimulating hormone, luteinizing hormone, and adrenocorticotropic hormone). Clinical confirmation that these adenomas did not exhibit excess hormone secretion was based on checking the patients' serum hormone levels and the absence of hormone-related symptoms. Cases showing positive immunohistochemical staining but not associated with clinical evidence of excess hormone secretion were diagnosed as NFPAs. Sufficient tissue for the construction of microarrays was available from 87 of the 124 cases. Clinical data, including age, sex, symptoms, recurrence, and survival status, were obtained via retrospective chart review.

\section{Radiographic analysis}

Tumor size and invasion status were evaluated using magnetic resonance imaging (MRI). Tumor size was recorded as the longest diameter. CSI was evaluated based on the criteria presented by Cottier et al. [26]. The samples were then classified into three groups: group I, neither SSE nor CSI; group II, only one of SSE or CSI; and group III, both SSE and CSI. We considered that a higher group level indicated a more aggressive tumor.

\section{Construction of tissue microarrays}

Four tissue microarray (TMA) blocks were constructed from archived, formalin-fixed paraffin blocks of the 87 samples with sufficient tumor cells. After checking the tumor cell-rich areas on the hematoxylin and eosin slides, a 3-mm-diameter core was collected from each sample and then arranged on premade recipient paraffin blocks (UB06-3, UNITMA, Seoul, Korea).

\section{Immunohistochemistry}

Sections ( $5 \mu \mathrm{m}$ in thickness) were obtained from the four TMA blocks described above. Immunohistochemical staining for PTTG1, E-cadherin, Ki-67, PITX2, and galectin-3 was performed using an automated slide-processing system (BenchMark XT, Ventana Medical Systems, Tucson, AZ, USA). The cut sections were pretreated with Cell Conditioner 1 (CC1, \#950124, Ventana Medical Systems) for 40 minutes. The sections were then incubated with PTTG1 antibody (1:200, \#34-1500, Thermo Fisher Scientific, Waltham, MA, USA), PITX2 antibody (1:50, \#ab32832, Abcam, Cambridge, UK), galectin-3 antibody (1:200, \#18-0393, Thermo Fisher Scientific), E-cadherin antibody (1:400, \#M3612, Dako, Glostrup, Denmark), and Ki-67 antibody (1:200, \#ab16667, Abcam) for 32 minutes each. The OptiView DAB Detection Kit (\#760-700, Ventana Medical Systems) was used for chromogenic detection.

Protein expression was evaluated by scoring the intensity of each stain on a scale of 0 (negative), 1 (weak), 2 (moderate), and 3 (strong). Since the proportion of each stain was uniform in most 
of the tumors, the expression level was determined only by the intensity of the stain. For Ki-67 staining, the stained cells were counted using a computer-assisted image analyzer (GenASIs HiPath, Applied Spectral Imaging Inc., Carlsbad, CA, USA).

\section{Statistical analysis}

The chi-squared test was used to determine the differences among expression groups of PTTG1, PITX2, galectin-3, and Ecadherin associated with clinical characteristics (e.g., SSE, CSI, sex, and recurrence). Linear-by-linear association was used to evaluate the correlation of the three invasiveness groups with PTTG1, PITX2, and galectin-3 expression levels. The independent t-test was used to analyze the relationships among the three candidate biomarkers and clinical factors including age, tumor size, and Ki-67 index. Analysis of variance was used to determine the differences between the three invasiveness groups associated with age and tumor size. An ordinal logistic regression model was used to compare the influences of individual factors, including PTTG1, PITX2, galectin-3, E-cadherin, Ki-67, age, sex, and tumor size, on NFPA invasiveness. All analyses were performed using IBM SPSS Statistics for Windows, ver. 25 (IBM Corp., Armonk, NY, USA). Statistical significance was set at $\mathrm{p}<0.05$.

\section{RESULTS}

\section{Patient characteristics}

The characteristics of the 87 patients with NFPA are shown in Table 1. The median age at diagnosis was 54 years (range, 16 to 83 years). Among these 87 patients, 48 (55.2\%) were men and $39(45.8 \%)$ were women. The mean tumor size $( \pm$ standard deviation [SD]) was $28.3 \pm 12.5 \mathrm{~mm}$, and there were two cases with missing tumor size data.

Overall, 77 patients (88.5\%) showed SSE and 33 cases (37.9\%) showed CSI on MRI (Table 1). Among the 87 patients, seven $(8.0 \%)$ were in group I, 50 (57.5\%) were in group II (SSE only, 47; CSI only, 3), and 30 (34.5\%) were in group III (Fig. 1).

Thirty-five patients (40.2\%) underwent post-operative radiotherapy. During each follow-up period (mean, 66 months; range, 0.5 to 209 months), tumor recurrences were observed in

Table 1. Clinical characteristics of patients with non-functioning pituitary adenomas

\begin{tabular}{ll}
\hline Clinical characteristic & No. (\%) \\
\hline Age (yr) & \\
$\quad$ Median (range) & $54(16-83)$ \\
Sex & \\
$\quad$ Male & $48(55.2)$ \\
$\quad$ Female & $39(44.8)$ \\
Tumor size (mm) & \\
$\quad$ Mean \pm SD & $28.3 \pm 12.5$ \\
Postoperative radiotherapy & \\
Yes & $35(40.2)$ \\
No & $52(59.8)$ \\
Recurrence & \\
Present & $16(18.4)$ \\
Absent & $71(81.6)$ \\
Suprasellar extension & \\
Present & $77(88.5)$ \\
Absent & $10(11.5)$ \\
Cavernous sinus invasion & \\
Present & $33(37.9)$ \\
Absent & $54(62.1)$ \\
\hline
\end{tabular}

$\mathrm{SD}$, standard deviation.

aUnknown tumor size in two cases.

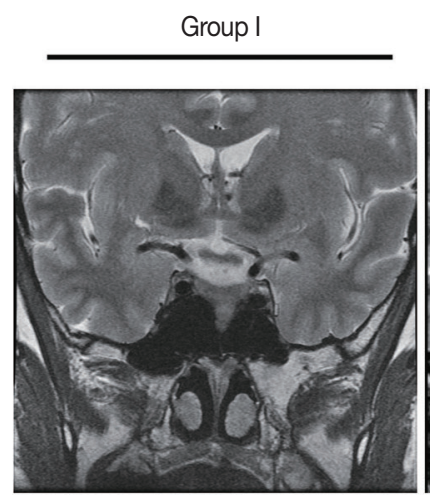

SSE (-) and CSI (-) $\mathrm{n}=7$

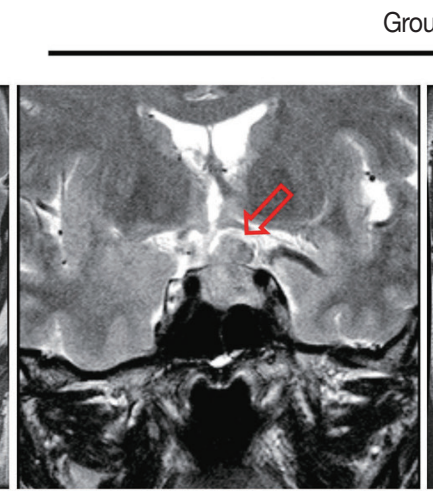

SSE (+) and CSI (-) $\mathrm{n}=47$
Group II

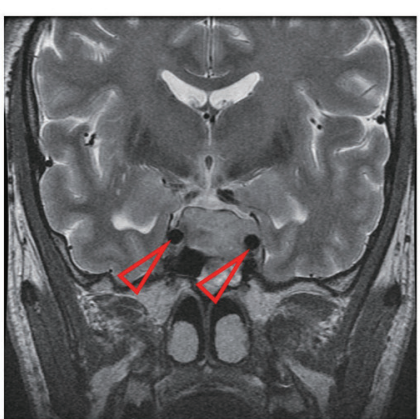

SSE (-) and CSI (+) $\mathrm{n}=3$

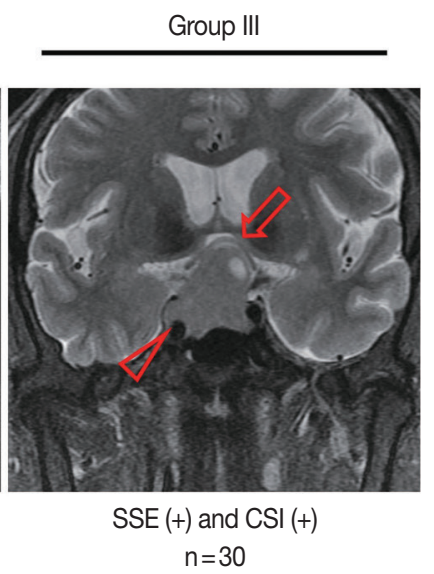

Fig. 1. Invasiveness groups of non-functioning pituitary adenoma (NFPA) based on suprasellar extension (SSE) and cavernous sinus invasion (CSI) on magnetic resonance imaging (MRI). The representative MRI images of SSE (arrows) and CSI (arrowheads) are shown. NFPAs were classified into three groups based on the MRI findings: group I, neither SSE nor CSI; group II, only one of SSE or CSI; group III, both SSE and CSI. 
16 patients (18.4\%), and non-disease-related death was observed in one patient. Disease-related death was not observed.

\section{Expression levels of PTTG1, PITX2, and galectin-3 and their relationship with invasiveness of NFPA}

The individual staining patterns and scores of PTTG1, PITX2, and galectin-3 are shown in Fig. 2. Staining of PTTG1 was observed in a cytoplasmic and paranuclear pattern. In PTTG1 staining, a score of 0 was considered no expression, scores of 1 and 2 were considered low expression, and a score of 3 was considered high expression. Staining of PITX2 and galectin-3 showed both nuclear and cytoplasmic patterns. In PITX2 and galectin-3 staining, a score of 0 was considered no expression, a score of 1 was considered low expression, and scores of 2 and 3 were considered high expression. Expression levels of PITX2 and galectin-3 were applied differently from PTTG1 because there were too few cases with scores of 3 in PITX2 and galectin-3 staining, making it difficult to calculate the appropriate statistics. Ten (11.5\%) specimens were negative for PTTG1, and $52(59.8 \%)$ and $25(28.7 \%)$ specimens showed low and high PTTG1 expression, respectively. In PITX2 staining, 21 (24.1\%) of the specimens showed no expression, 30 (34.5\%) showed low

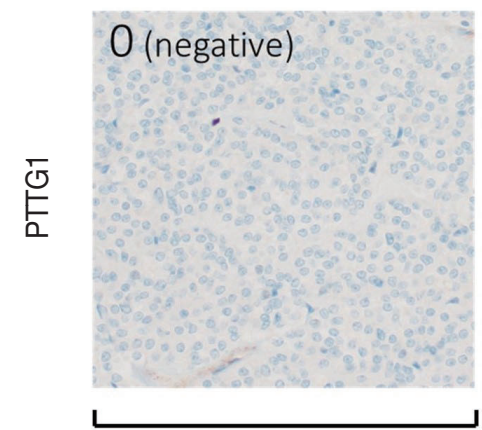

Negative $(n=10)$

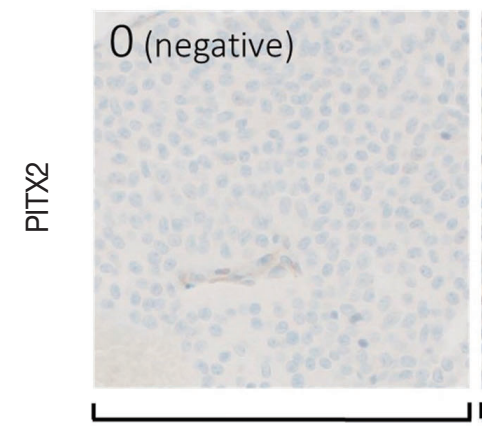

Negative $(n=21)$

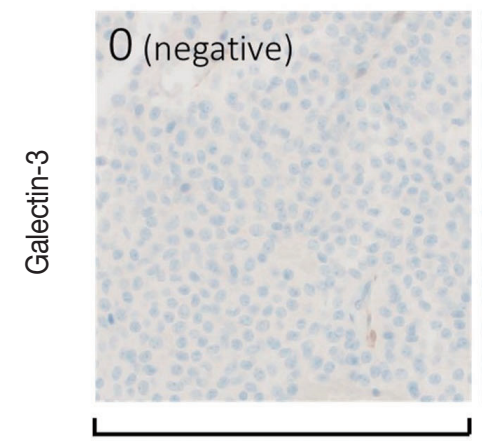

Negative $(n=37)$

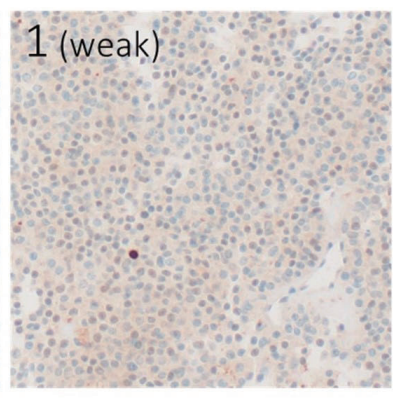

Low $(n=52)$

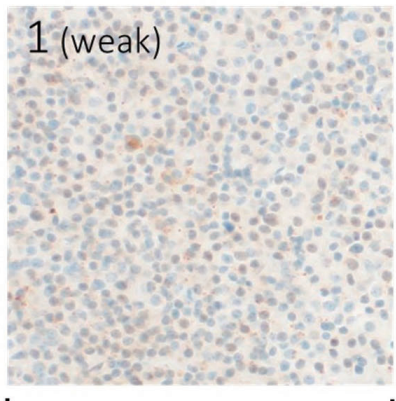

Low $(n=30)$

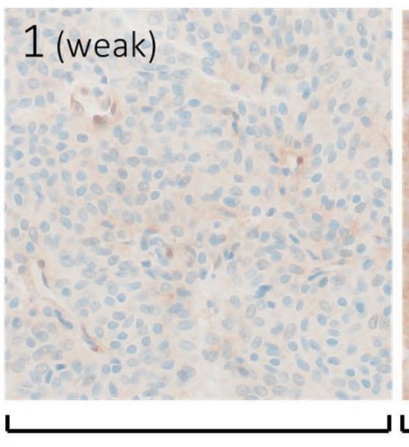

Low $(n=27)$
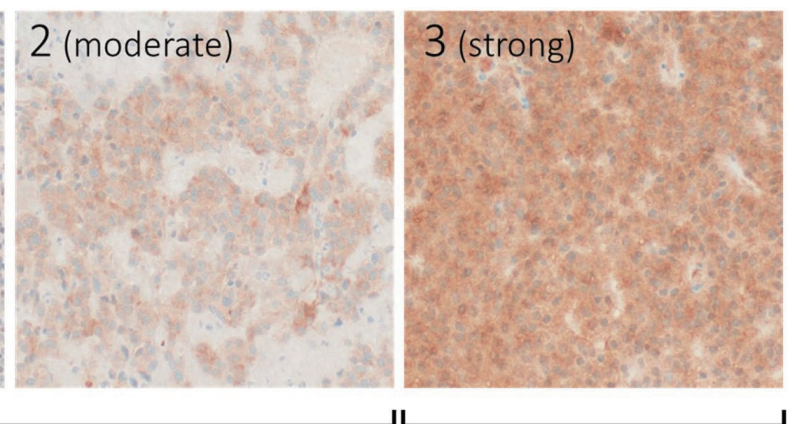

High $(n=25)$

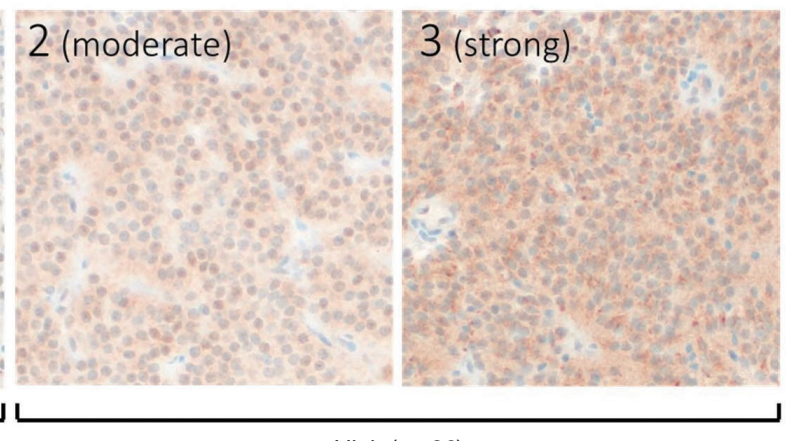

High $(n=36)$

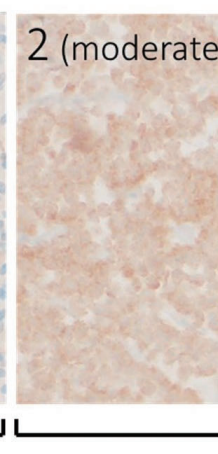

\section{Cans}

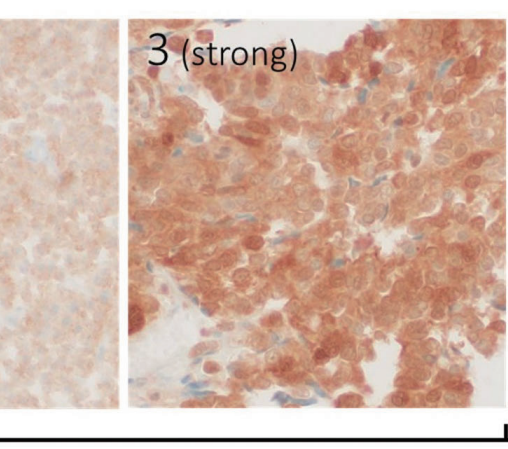

$\operatorname{High}(\mathrm{n}=23)$

Fig. 2. Scoring of immunohistochemical staining for pituitary tumor transforming gene (PTTG1), paired-like homeodomain 2 (PITX2), and galectin-3. The intensity of immunohistochemical stains was scored from 0 (negative) to 3 (strong). PTTG1: a score of 0 was regarded as negative, scores of 1 and 2 were regarded as low, and a score of 3 was regarded as high expression. PITX2 and galectin-3: a score of 0 was regarded as negative, a score of 1 was regarded as low, and scores of 2 and 3 were regarded as high expression. 
expression, and $36(41.4 \%)$ showed high expression. In galectin-3 staining, 37 (42.5\%) specimens showed no expression, 27 $(31.0 \%)$ showed low expression, and 23 (26.4\%) showed high expression.

PTTG1 expression was significantly correlated with NFPA invasiveness. In the high PTTG1 expression group, SSE or CSI was observed more frequently than in the negative or low PTTG1 expression group ( $\mathrm{p}=.033$ ) (Table 2). Similarly, the higher invasiveness group showed higher PTTG1 expression, and the lower invasiveness group showed lower PTTG1 expression $(\mathrm{p}=.003)$ (Table 3, Fig. 3). There was no association between PITX2 or galectin-3 expression and invasiveness (Table 3). As described above, expression levels of PITX2 and galectin-3 were applied differently from PTTG1 because the number of cases with scores of 3 in PITX2 and galectin-3 was too few (score 1, low expression; scores 2 and 3 , high expression). Nevertheless, even when the expression levels of PITX2 and galectin- 3 were classified in the same way as PTTG1 expression (scores 1 and 2, low expression; score 3 , high expression), there was no statistically signifi-

Table 2. Clinicopathologic characteristics according to PTTG1 expression

\begin{tabular}{|c|c|c|c|}
\hline Variable & $\begin{array}{l}\text { Negative or low } \\
\text { PTTG1 (n=62) }\end{array}$ & $\begin{array}{l}\text { High PTTG1 } \\
\quad(n=25)\end{array}$ & $p$-value \\
\hline Age (yr) & & & .656 \\
\hline Median (range) & $53(31-83)$ & $55(16-83)$ & \\
\hline Sex & & & .183 \\
\hline Male & $37(59.7)$ & $11(44.0)$ & \\
\hline Female & $25(40.3)$ & $14(56.0)$ & \\
\hline \multicolumn{4}{|l|}{ Invasiveness status } \\
\hline Suprasellar extension & & & $.033^{\mathrm{a}}$ \\
\hline Present & $52(83.9)$ & $25(100)$ & \\
\hline Absent & $10(16.1)$ & 0 & \\
\hline Cavernous sinus invasion & & & $.027^{\mathrm{a}}$ \\
\hline Present & $19(30.6)$ & $14(56.0)$ & \\
\hline Absent & $43(69.4)$ & $11(44.0)$ & \\
\hline Tumor size $(\mathrm{mm})^{\mathrm{b}}$ & & & .612 \\
\hline Mean $\pm S D$ & $29.0 \pm 13.5$ & $26.5 \pm 9.2$ & \\
\hline \multicolumn{4}{|l|}{ Recurrence } \\
\hline Present & $13(21.0)$ & $3(12.0)$ & .329 \\
\hline Absent & 49 (79.0) & $22(88.0)$ & \\
\hline Present (N-PRT) & $11(28.9)$ & $0(0.0)$ & .103 \\
\hline Absent (N-PRT) & $27(71.1)$ & $14(100.0)$ & \\
\hline E-cadherin & & & .183 \\
\hline Positive (score 1-3) & $25(40.3)$ & $14(56.0)$ & \\
\hline Negative (score 0) & $37(59.7)$ & $11(44.0)$ & \\
\hline Ki-67 index (\%) & & & .389 \\
\hline Mean \pm SD & $1.3 \pm 1.1$ & $1.8 \pm 2.5$ & \\
\hline
\end{tabular}

Values are presented as number (\%) unless otherwise indicated.

PTTG1, pituitary tumor transforming gene 1; SD, standard deviation; N-PRT, no post-operative radiotherapy.

aStatistically significant $(p<.05)$; bunknown tumor size in two cases with low PTTG1 expression. cant correlation between PITX2 and galectin-3 expression and NFPA invasiveness ( $\mathrm{p}=.271$ for PITX2, $\mathrm{p}=.997$ for galectin-3).

PTTG1, PITX2, and galectin-3 did not show any relationship with other clinical characteristics, such as age, sex, tumor size, and recurrence. Even in the group without post-operative radiotherapy, there was no correlation between PTTG1 expression and recurrence (Table 2).

\section{Relationships of E-cadherin loss and Ki-67 index with PTTG1 expression}

The individual staining patterns of E-cadherin and $\mathrm{Ki}-67$ are shown in Fig. 4. The E-cadherin stained in a membranous pattern. A score of 0 was considered negative, and scores of 1 to 3 were considered positive for E-cadherin staining. Forty-eight $(55.2 \%)$ of the specimens were negative, and $39(44.8 \%)$ showed positive E-cadherin expression. In the high PTTG1 expression group, $11(44.0 \%)$ showed negative E-cadherin expression. In the negative or low PTTG1 expression group, 37 (59.7\%) showed negative E-cadherin expression (Table 2). There was no significant correlation between the expression of E-cadherin and PTTG1.

Table 3. Clinicopathologic characteristics according to tumor invasiveness group

\begin{tabular}{|c|c|c|c|c|}
\hline \multirow{2}{*}{ Variable } & \multicolumn{3}{|c|}{ Tumor invasion group } & \multirow{2}{*}{$p$-value } \\
\hline & $I(n=7)$ & $\|(n=50)$ & III $(n=30)$ & \\
\hline Age (yr) & & & & .605 \\
\hline Median (range) & $50(42-71)$ & $55(16-83)$ & $54.5(31-83)$ & \\
\hline $\operatorname{Sex}(\%)$ & & & & .790 \\
\hline Male & $3(42.9)$ & $28(56.0)$ & $17(56.7)$ & \\
\hline Female & $4(57.1)$ & $22(44.0)$ & $13(43.3)$ & \\
\hline Tumor size $(\mathrm{mm})^{\mathrm{a}}$ & & & & $<.001^{b}$ \\
\hline Mean $\pm S D$ & $17.2 \pm 5.1$ & $25.9 \pm 9.2$ & $35.3 \pm 15.0$ & \\
\hline PTTG1 (\%) & & & & $.013^{b}$ \\
\hline High & 0 & $11(22.0)$ & $14(46.7)$ & \\
\hline Negative or low & $7(100)$ & $39(78.0)$ & $16(53.3)$ & \\
\hline PITX2 (\%) & & & & .745 \\
\hline High & $3(42.9)$ & $19(38.0)$ & $14(46.7)$ & \\
\hline Low or negative & $4(57.1)$ & $31(62.0)$ & 16 (53.3) & \\
\hline Galectin-3 (\%) & & & & .605 \\
\hline High & $1(14.3)$ & $15(30.0)$ & $7(23.3)$ & \\
\hline Low or negative & $6(85.7)$ & $35(70.0)$ & $23(76.7)$ & \\
\hline E-cadherin (\%) & & & & .989 \\
\hline Positive & $3(42.9)$ & $23(46.0)$ & $13(43.3)$ & \\
\hline Negative & $4(57.1)$ & $27(54.0)$ & $17(56.7)$ & \\
\hline Ki-67 index (\%) & & & & .681 \\
\hline Mean $\pm S D$ & $1.5 \pm 0.8$ & $1.6 \pm 2.0$ & $1.2 \pm 1.1$ & \\
\hline
\end{tabular}

Values are presented as number (\%) unless otherwise indicated.

SD, standard deviation; PTTG1, pituitary tumor transforming gene 1; PITX2, paired-like homeodomain 2.

aUnknown tumor size in two cases in group III; 'btatistically significant $(p<$ .05). 

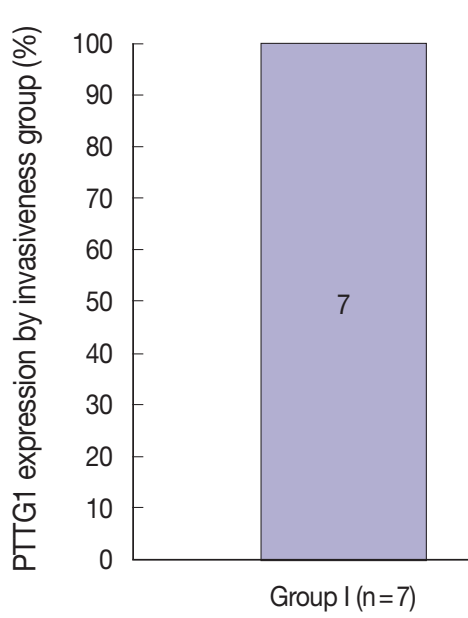

High PTTG1 $\square$ Negative of low PTTG1

Fig. 3. Stacked column chart showing the ratio of pituitary tumor transforming gene (PTTG1) expression in each invasiveness group. As the level of invasiveness increased, the proportion of high PTTG1 expression tended to increase.

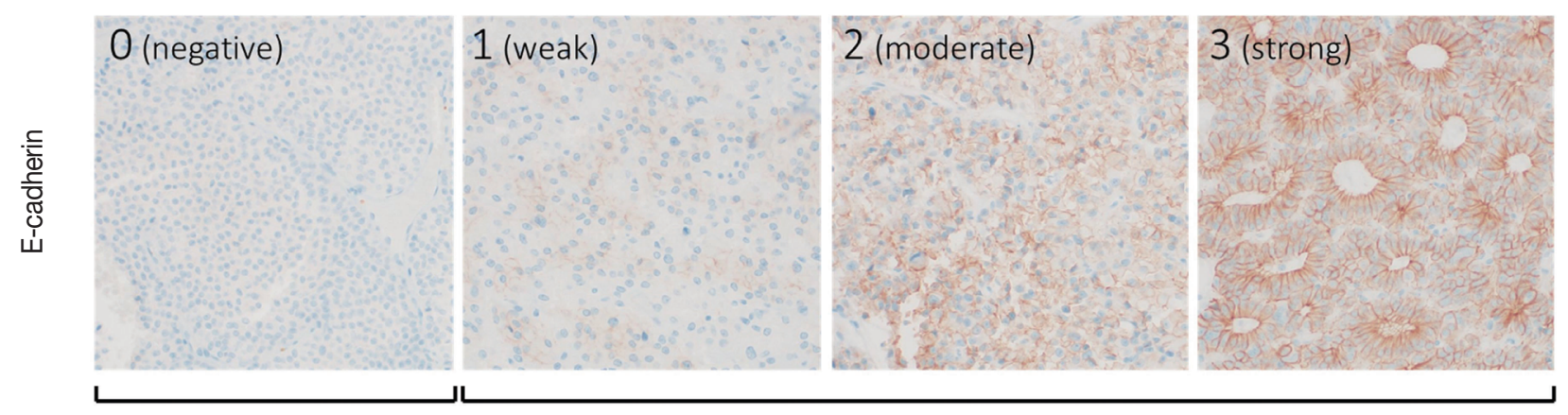

Negative $(n=48)$

Positive $(n=39)$

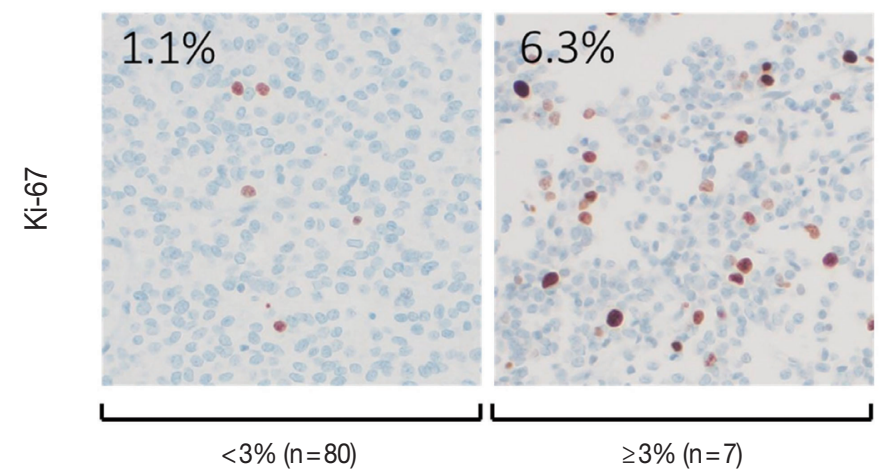

Fig. 4. Scoring of immunohistochemical staining for E-cadherin and Ki-67. E-cadherin: a score of 0 was regarded as negative, and scores of 1 to 3 were regarded as positive expression. Ki-67 index was measured by image analyzer, and two samples of the stain are shown.

The Ki-67 staining showed nuclear staining, and the overall average index $( \pm \mathrm{SD})$ was $1.4 \% \pm 1.4 \%$. The average $\mathrm{Ki}-67$ index in the high PTTG1 expression group was $1.8 \% \pm 2.5 \%$, which was higher than that observed in the negative or low PTTG1 expression group $(1.3 \% \pm 1.1 \%)$ (Table 2$)$. Ki-67 expression tended to be associated with PTTG1 expression; however, this result was not statistically significant $(\mathrm{p}=.389)$.

\section{Other clinical factors associated with aggressive features of NFPA}

The tumor invasiveness was significantly correlated with the tumor size: the higher the level of the invasion group, the larger the tumor size $(\mathrm{p}<.001)$ (Table 3, Fig. 5A). In addition, tumor size was associated with the recurrence of NFPA (Fig. 5B). As the size of the tumor increased, the NFPA tended to recur. How- 

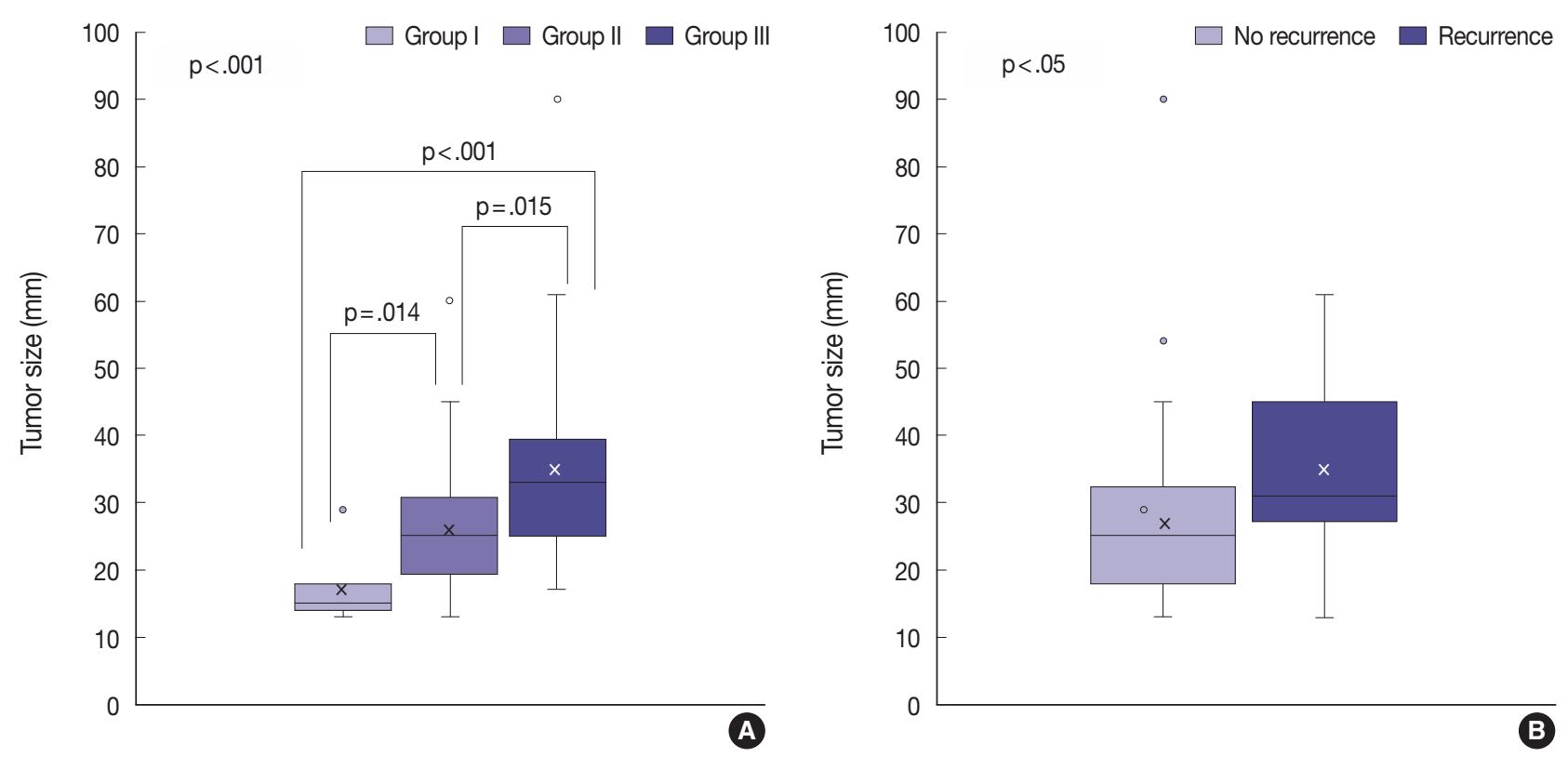

Fig. 5 . Box plots of the distribution of the tumor size by invasion group and recurrence. A larger tumor size was associated with higher invasiveness (A) and a higher recurrence rate $(B)$.

Table 4. Multivariate analysis (ordinal logistic regression test) of invasiveness groups associated with clinicopathologic variables

\begin{tabular}{lccc}
\hline Variable & Estimate & $\mathrm{p}$-value & 95\% Confidence interval \\
\hline Age & 0.030 & .180 & -0.014 to 0.074 \\
Sex & -0.085 & .876 & -1.152 to 0.983 \\
Tumor size & 0.13 & $<.001^{\mathrm{a}}$ & 0.069 to 0.191 \\
PTTG1 & 2.518 & $.001^{\mathrm{a}}$ & 1.086 to 3.950 \\
PITX2 & -0.320 & .391 & -1.052 to 0.411 \\
Galectin-3 & 0.195 & .575 & -0.485 to 0.875 \\
E-cadherin & 0.482 & .498 & -0.914 to 1.879 \\
Ki-67 index & 0.347 & .696 & -1.398 to 2.093 \\
\hline
\end{tabular}

PTTG1, pituitary tumor transforming gene 1; PITX2, paired-like homeodomain 2.

aStatistically significant $(p<.05)$.

ever, post-operative radiotherapy was not considered in this study.

\section{Multivariate analysis of NFPA invasiveness}

Multivariate analysis using ordinal logistic regression showed that PTTG1 expression and tumor size were statistically associated with tumor invasiveness levels $(\mathrm{p}=.001$ for PTTG1, $\mathrm{p}<$ .001 for tumor size) (Table 4). The other variables including PITX2, galectin-3, E-cadherin, Ki-67, age, and sex did not show any relationships with the tumor invasiveness groups. In addition, the PTTG1 expression and the tumor size were statistically independent of each other and of other variables including PITX2, galectin-3, E-cadherin, Ki-67, age, and sex (Table 4).

\section{DISCUSSION}

Although several studies have shown PTTG1 overexpression in pituitary adenomas and in tumors of other organs, a limited number of studies have investigated the relationship between PTTG1 expression and tumor invasiveness $[18,27,28]$. A metaanalysis of these studies concluded that there was a significant relationship between PTTG1 expression and the invasiveness of pituitary adenomas [29]. However, most of these studies were limited to a specific subtype of functioning pituitary adenomas or did not distinguish functioning adenomas from non-functioning adenomas.

In this study, we performed an analysis limited to NFPA, and demonstrated that PTTG1 expression was significantly correlated with the invasiveness of NFPA. Only a few previous studies have explored PTTG1 expression and invasiveness status limited to NFPA $[18,27,30]$. A study by Trott et al. [30] revealed that PTTG1 expression and the invasiveness status of NFPA had an important correlation. Additionally, research by McCabe et al. [18] revealed PTTG1 overexpression in both invasive NFPAs and invasive functioning pituitary adenomas. The results of the present study support these two previous studies. In contrast, a previous study conducted by Zhang et al. [27] did not identify a correlation between PTTG1 expression and invasiveness in NFPA but did reveal the correlation in functioning pituitary adenomas. However, the number of the NFPA samples in the study by Zhang et al. [27] was 30, which was smaller than those 
included in the studies that confirmed the significant correlation between PTTG1 and NFPA invasiveness, including our study; the numbers of NFPA samples were 56 for the study by Trott et al. [30], 92 for the study by McCabe et al. [18], and 87 for the present study. It is therefore reasonable to assume that PTTG1 expression is correlated with NFPA invasiveness, as it is in other organ tumors.

Several studies revealed a correlation between PTTG1 expression and the recurrence of pituitary adenomas [30-32]. One of these studies by Fillippella et al. [31] did not report the factor of postoperative radiotherapy. Additionally, other studies by Trott et al. [30] and Noh et al. [32] excluded the patients who underwent postoperative radiotherapy. The present study did not reveal a correlation between PTTG1 expression and recurrence of the tumor. We also analyzed PTTG1 expression and recurrence in a group of patients who did not undergo post-operative radiotherapy, but no meaningful results were obtained, contrary to the results of previous studies.

A few studies have demonstrated that PITX2 expression is increased in pituitary adenomas, highlighting a correlation between PITX2 overexpression and the aggressiveness of NFPAs $[33,34]$. In comparison, however, the results of the present study did not show a relationship between PITX2 expression and NFPA invasiveness. These contradictory results are probably due to the small number of cases in our study. However, since few studies have been conducted on this subject to date, more data should be acquired to establish the relationship between PITX2 expression and NFPA invasiveness.

In pituitary adenoma, galectin-3 expression is increased in functioning pituitary adenomas, especially in functioning corticotroph adenomas, but not in NFPAs [35,36]. In addition, there have been several studies on galectin- 3 and the aggressive behavior of pituitary adenoma, which demonstrated that galectin-3 expression is positively correlated with the aggressiveness of pituitary adenomas, but not in NFPAs [36,37]. In the present study with only NFPAs, the intensity of galectin-3 staining was varied, and there was no correlation with the invasiveness of NFPAs. Although we noted a high intensity of galectin-3 staining in our study, the validity of this result should be reassessed because we did not compare the intensity in NFPAs with that in functioning adenomas.

As PTTG1 was the only protein that showed an association with the invasiveness of NFPAs in this study, further investigation was performed on the relationship among PTTG1, E-cadherin, and Ki-67. Based on the previous studies, which showed an association between PTTG1 and E-cadherin loss in the process of EMT in other organs (e.g., head and neck, esophagus, ovary, and breast), we hypothesized that E-cadherin is correlated with PTTG1 expression in NFPAs; this is the first study on PTTG1 and E-cadherin in pituitary adenoma $[19,22,23]$. However, we could not identify the relationship between PTTG1 expression and E-cadherin loss in NFPAs. This may be due to the small number of cases, but it may also be the result of PTTG1 not being the main causative factor for E-cadherin loss. There are several epigenetic mechanisms that induce E-cadherin loss other than PTTG1, including some microRNAs (e.g., miR-192, miR200, and miR-205) and the hypermethylation of $C D H 1$ promoter, a gene encoding E-cadherin [38,39]. The results of our study suggest that the factors such as the microRNAs and the hypermethylation of $C D H 1$ promoter may play a greater role than PTTG1 in inducing E-cadherin loss in pituitary adenomas.

There have been previous studies on PTTG1 expression and cell proliferation, but the results are conflicting [40-43]. Studies by Heaney et al. [40] and Wang et al. [41] revealed that PTTG1 promoted cell proliferation. However, studies by Mu et al. [42] and Yu et al. [43] revealed that there was no correlation between PTTG1 and cell proliferation, which is consistent with the results of the present study. In terms of its ability to inhibit chromatid separation during mitosis, PTTG1 is expected to inhibit cell proliferation, but its ability to induce angiogenesis or distupt the DNA repair system may promote cell proliferation. As there are contradictory views in many studies, the relationship between PTTG1 expression and cell proliferation needs to be further studied.

Additionally, we determined that the invasiveness status and recurrence of NFPAs were significantly correlated with tumor size. According to many studies, pituitary adenomas show aggressive behavior as the size of the tumor increases, which is consistent with the results of our study [44]. However, with respect to tumor recurrence, previous studies have shown that tumor recurrence is not influenced by tumor size, which contradicts the results of the present study [45]. Although the results of our study revealed a correlation between tumor size and recurrence, it should be noted that the factor of post-operative radiotherapy was not considered.

In conclusion, we demonstrated here that PTTG1 has the potential to be a predictive marker for the invasiveness of NFPAs. Although there are many previous studies that have explored the relationship between PTTG1 expression and tumors including pituitary adenoma, in the present study, we elucidated the role of PTTG1, particularly in NFPAs, for the first time. Furthermore, we provide evidence for the development of PTTG1-tar- 
geted agents and references for studies on the correlation between PTTG1 and various tumors, which have been continually reported.

\section{Ethics Statement}

This study was approved by the Institutional Review Board of the Keimyung University Dongsan Medical Center, with waiver of informed consent (DSMC 2021-02-036).

\section{Availability of Data and Material}

The datasets generated or analyzed during the study are available from the corresponding author on reasonable request.

\section{Code Availability}

Not applicable.

\section{ORCID}

Su Jung Kum Hye Won Lee Soon Gu Kim Hyungsik Park Ilseon Hwang Sang Pyo Kim

https://orcid.org/0000-0003-4871-3707 https://orcid.org/0000-0001-8540-524X https://orcid.org/0000-0002-1436-8442 https://orcid.org/0000-0001-5476-3853 https://orcid.org/0000-0002-6122-4417 https://orcid.org/0000-0003-0948-2408

\section{Author Contributions}

Conceptualization: SPK. Data curation: SJK, IH. Formal analysis: IH, SGK, SJK. Investigation: SJK, HP. Methodology: SPK, SJK. Resources: HWL. Supervision: SPK, IH. Visualization: SJK, IH. Writing—original draft: SJK, IH. Writing-review \& editing: SJK, SPK, IH, HWL. Approval of final manuscript: all authors.

\section{Conflicts of Interest}

The authors declare that they have no potential conflicts of interest.

\section{Funding Statement}

This work was supported by the research promoting grant from the Institute for Cancer Reseach Keimyung University Dongsan Medical Center in 2018.

\section{References}

1. Gadelha MR, Trivellin G, Hernandez Ramirez LC, Korbonits M. Genetics of pituitary adenomas. Front Horm Res 2013; 41: 111-40.

2. Greenman Y, Cooper O, Yaish I, et al. Treatment of clinically nonfunctioning pituitary adenomas with dopamine agonists. Eur J Endocrinol 2016; 175: 63-72.

3. Greenman Y, Tordjman K, Osher E, et al. Postoperative treatment of clinically nonfunctioning pituitary adenomas with dopamine agonists decreases tumour remnant growth. Clin Endocrinol (Oxf) 2005; 63: 39-44.

4. DeLellis RA, Lloyd RV, Heitz PU, Eng C. World Health Organization classification of tumours: pathology and genetics of tumours of endocrine organs. Lyons: IARC Press, 2004.

5. Lloyd RV, Osamura RY, Kloppel G, Rosai J. WHO cassification of tumours of endocrine organs. Lyons: IARC Press, 2012; 11-3.

6. Saeger W, Ludecke DK, Buchfelder M, Fahlbusch R, Quabbe HJ, Petersenn S. Pathohistological classification of pituitary tumors: 10 years of experience with the German Pituitary Tumor Registry. Eur
J Endocrinol 2007; 156: 203-16.

7. Zada G, Woodmansee WW, Ramkissoon S, Amadio J, Nose V, Laws ER Jr. Atypical pituitary adenomas: incidence, clinical characteristics, and implications. J Neurosurg 2011; 114: 336-44.

8. Scoazec JY, Couvelard A, Reseau T. Classification of pancreatic neuroendocrine tumours: changes made in the 2017 WHO classification of tumours of endocrine organs and perspectives for the future. Ann Pathol 2017; 37: 444-56.

9. Trouillas J, Roy P, Sturm N, et al. A new prognostic clinicopathological classification of pituitary adenomas: a multicentric casecontrol study of 410 patients with 8 years post-operative follow-up. Acta Neuropathol 2013; 126: 123-35.

10. Matsuyama J. Ki-67 expression for predicting progression of postoperative residual pituitary adenomas: correlations with clinical variables. Neurol Med Chir (Tokyo) 2012; 52: 563-9.

11. Chiloiro S, Doglietto F, Trapasso B, et al. Typical and atypical pituitary adenomas: a single-center analysis of outcome and prognosis. Neuroendocrinology 2015; 101: 143-50.

12. Kim JS, Lee YS, Jung MJ, Hong YK. The predictive value of pathologic features in pituitary adenoma and correlation with pituitary adenoma recurrence. J Pathol Transl Med 2016; 50: 419-25.

13. Mooney MA, Hardesty DA, Sheehy JP, et al. Rater reliability of the hardy classification for pituitary adenomas in the magnetic resonance imaging era. J Neurol Surg B Skull Base 2017; 78: 413-8.

14. Thapar K, Scheithauer BW, Kovacs K, Pernicone PJ, Laws ER Jr. p53 expression in pituitary adenomas and carcinomas: correlation with invasiveness and tumor growth fractions. Neurosurgery 1996; 38: 765-70.

15. Oliveira MC, Marroni CP, Pizarro CB, Pereira-Lima JF, BarbosaCoutinho LM, Ferreira NP. Expression of p53 protein in pituitary adenomas. Braz J Med Biol Res 2002; 35: 561-5.

16. Salehi F, Agur A, Scheithauer BW, Kovacs K, Lloyd RV, Cusimano M. Biomarkers of pituitary neoplasms: a review (Part II). Neurosurgery 2010; 67: 1790-8.

17. Sav A, Rotondo F, Syro LV, Scheithauer BW, Kovacs K. Biomarkers of pituitary neoplasms. Anticancer Res 2012; 32: 4639-54.

18. McCabe CJ, Khaira JS, Boelaert K, et al. Expression of pituitary tumour transforming gene (PTTG) and fibroblast growth factor-2 (FGF-2) in human pituitary adenomas: relationships to clinical tumour behaviour. Clin Endocrinol (Oxf) 2003; 58: 141-50.

19. Shah PP, Kakar SS. Pituitary tumor transforming gene induces epithelial to mesenchymal transition by regulation of Twist, Snail, Slug, and E-cadherin. Cancer Lett 2011; 311: 66-76.

20. Zou H, McGarry TJ, Bernal T, Kirschner MW. Identification of a vertebrate sister-chromatid separation inhibitor involved in transformation and tumorigenesis. Science 1999; 285: 418-22.

21. Salehi F, Kovacs K, Scheithauer BW, Lloyd RV, Cusimano M. Pituitary tumor-transforming gene in endocrine and other neoplasms: a review and update. Endocr Relat Cancer 2008; 15: 721-43.

22. Feng W, Xiaoyan X, Shenglei L, Hongtao L, Guozhong J. PTTG1 cooperated with GLI1 leads to epithelial-mesenchymal transition in esophageal squamous cell cancer. Oncotarget 2017; 8: 92388-400.

23. Yoon CH, Kim MJ, Lee H, et al. PTTG1 oncogene promotes tumor malignancy via epithelial to mesenchymal transition and expansion of cancer stem cell population. J Biol Chem 2012; 287: 19516-27.

24. Nangia-Makker P, Honjo Y, Sarvis R, et al. Galectin-3 induces endothelial cell morphogenesis and angiogenesis. Am J Pathol 2000; 156: 899-909. 
25. Yoshii T, Fukumori T, Honjo Y, Inohara H, Kim HR, Raz A. Galectin-3 phosphorylation is required for its anti-apoptotic function and cell cycle arrest. J Biol Chem 2002; 277: 6852-7.

26. Cottier JP, Destrieux C, Brunereau L, et al. Cavernous sinus invasion by pituitary adenoma: MR imaging. Radiology 2000; 215: 463-9.

27. Zhang X, Horwitz GA, Heaney AP, et al. Pituitary tumor transforming gene (PTTG) expression in pituitary adenomas. J Clin Endocrinol Metab 1999; 84: 761-7.

28. Raverot G, Wierinckx A, Dantony E, et al. Prognostic factors in prolactin pituitary tumors: clinical, histological, and molecular data from a series of 94 patients with a long postoperative follow-up. J Clin Endocrinol Metab 2010; 95: 1708-16.

29. Li Y, Zhou LP, Ma P, et al. Relationship of PTTG expression with tumor invasiveness and microvessel density of pituitary adenomas: a meta-analysis. Genet Test Mol Biomarkers 2014; 18: 279-85.

30. Trott G, Ongaratti BR, de Oliveira Silva CB, et al. PTTG overexpression in non-functioning pituitary adenomas: Correlation with invasiveness, female gender and younger age. Ann Diagn Pathol 2019; 41: 83-9.

31. Filippella M, Galland F, Kujas M, et al. Pituitary tumour transforming gene (PTTG) expression correlates with the proliferative activity and recurrence status of pituitary adenomas: a clinical and immunohistochemical study. Clin Endocrinol (Oxf) 2006; 65: 536-43.

32. Noh TW, Jeong HJ, Lee MK, Kim TS, Kim SH, Lee EJ. Predicting recurrence of nonfunctioning pituitary adenomas. J Clin Endocrinol Metab 2009; 94: 4406-13.

33. Tamura R, Ohara K, Morimoto Y, et al. PITX2 expression in nonfunctional pituitary neuroendocrine tumor with cavernous sinus invasion. Endocr Pathol 2019; 30: 81-9.

34. Acunzo J, Roche C, Defilles C, et al. Inactivation of PITX2 transcription factor induced apoptosis of gonadotroph tumoral cells. Endocrinology 2011; 152: 3884-92.

35. Jin L, Riss D, Ruebel K, et al. Galectin-3 expression in functioning and silent ACTH-producing adenomas. Endocr Pathol 2005; 16:
$107-14$.

36. Zhang Y, He N, Zhou J, Chen Y. The relationship between MRI invasive features and expression of EMMPRIN, galectin-3, and microvessel density in pituitary adenoma. Clin Imaging 2011; 35: 165-73.

37. Righi A, Morandi L, Leonardi E, et al. Galectin-3 expression in pituitary adenomas as a marker of aggressive behavior. Hum Pathol 2013; 44: 2400-9.

38. Qian ZR, Sano T, Yoshimoto K, et al. Tumor-specific downregulation and methylation of the $\mathrm{CDH} 13$ (H-cadherin) and CDH1 (Ecadherin) genes correlate with aggressiveness of human pituitary adenomas. Mod Pathol 2007; 20: 1269-77.

39. Wong TS, Gao W, Chan JY. Interactions between E-cadherin and microRNA deregulation in head and neck cancers: the potential interplay. Biomed Res Int 2014; 2014: 126038.

40. Heaney AP, Horwitz GA, Wang Z, Singson R, Melmed S. Early involvement of estrogen-induced pituitary tumor transforming gene and fibroblast growth factor expression in prolactinoma pathogenesis. Nat Med 1999; 5: 1317-21.

41. Wang Z, Moro E, Kovacs K, Yu R, Melmed S. Pituitary tumor transforming gene-null male mice exhibit impaired pancreatic beta cell proliferation and diabetes. Proc Natl Acad Sci U S A 2003; 100: 3428-32

42. Mu YM, Oba K, Yanase T, et al. Human pituitary tumor transforming gene (hPTTG) inhibits human lung cancer A549 cell growth through activation of p21(WAF1/CIP1). Endocr J 2003; 50: 771-81.

43. Yu R, Cruz-Soto M, Li Calzi S, Hui H, Melmed S. Murine pituitary tumor-transforming gene functions as a securin protein in insulinsecreting cells. J Endocrinol 2006; 191: 45-53.

44. Dekkers OM, Karavitaki N, Pereira AM. The epidemiology of aggressive pituitary tumors (and its challenges). Rev Endocr Metab Disord 2020; 21: 209-12.

45. Roelfsema F, Biermasz NR, Pereira AM. Clinical factors involved in the recurrence of pituitary adenomas after surgical remission: a structured review and meta-analysis. Pituitary 2012; 15: 71-83. 